\title{
Factors predicting success of trial of labour after caesarean in current scenario
}

\author{
Pooja S. Singh*, Jaishree Bamaniya, Ami Shah
}

\begin{abstract}
Associate Professor, Department of Obstetrics and Gynaecology, GCS Medical College and Hospital, Ahmedabad,
\end{abstract} Gujarat, India

Received: 28 August 2016

Accepted: 27 September 2016

*Correspondence:

Dr. Pooja S. Singh,

E-mail:drpsomesh03@yahoo.co.in

Copyright: (c) the author(s), publisher and licensee Medip Academy. This is an open-access article distributed under the terms of the Creative Commons Attribution Non-Commercial License, which permits unrestricted non-commercial use, distribution, and reproduction in any medium, provided the original work is properly cited.

\begin{abstract}
Background: Cesarean deliveries have been on the rise in recent decades, and as a result the question of how to manage subsequent deliveries becomes ever more important. The National Institute for Health and Care Excellence, RCOG, American College of Obstetricians and Gynecologists, and National Institutes of Health are in consensus that planned VBAC is a clinically safe choice for the majority of women with a single previous lower segment caesarean delivery, the authors explain. Aim of the study was to identify potential predictors of successful VBAC and study the risk and benefits involved.

Methods: This was a prospective study in GCS Medical College. Seventy five cases were chosen fulfilling the eligibility criteria and were subjected to study.

Results: The outcome was measured as successful VBAC. Out of 75 cases included in our study, 43 cases $(57.3 \%$ of total cases) qualified for TOLAC during labour. Successful TOL (trial of labor) was observed in $48.8 \%$ (21 out of 43 ) resulting in VBAC (vaginal birth after caesarean) and the rest $51.2 \%$ of failed TOL ended in repeat caesarean delivery.

Conclusions: In our study, most of the women with successful VBAC were less than 30 years of age (95.2\%). In our study, successful VBAC cases (90.5\% of cases) presented with cervical dilatation more than equal to $4 \mathrm{~cm}$ and most cases $(95.2 \%)$ of VBAC delivered before 38 weeks of gestation. Out of all VBAC's, 62\% (13 out of 21) had ante natal indication for their previous caesarean delivery; most common indication being breech. In our study, Repeat C.S. in failed TOL was performed for scar dehiscence in $41 \%$ cases.
\end{abstract}

Keywords: ERCD, RPCS, TOLAC, VBAC

\section{INTRODUCTION}

The World Health Organization has stated that national cesarean rates greater than $10-15 \%$ indicate unnecessary maternal risk. Nevertheless the current cesarean rate in the US is $32.2 \%$ (CDC). With this high rate of cesarean delivery the question of the route of delivery for subsequent pregnancies becomes ever more important.

The National Institute for Health and Care Excellence, RCOG, American College of Obstetricians and
Gynaecologists, and National Institutes of Health are in consensus "that planned VBAC is a clinically safe choice for the majority of women with a single previous lower segment caesarean delivery," the authors explain. "Such a strategy is also supported by health economic modelling, and would also at least limit any escalation of the caesarean delivery rate and maternal morbidity associated with multiple caesarean deliveries."1 The controversy lies in the risk associated with unsuccessful VBAC, which is associated with an increased risk of uterine rupture and maternal mortality. 
Both the American College of Obstetricians and Gynaecologists (ACOG) and the National Institutes of Health (NIH) suggest that a trial of labour after cesarean (TOLAC) to attempt a vaginal birth after cesarean (VBAC) is an acceptable option for a woman who has undergone one prior cesarean delivery with a low transverse uterine incision, assuming there are no other conditions that would normally require a cesarean delivery (as an example, placenta previa). ${ }^{2,3}$

In August 2010, the College issued a new Practice Bulletin, Vaginal Birth after Previous Cesarean Delivery, which states that attempting a VBAC is a safe and appropriate choice for most women who have had a prior cesarean delivery, including for some women who have had two previous cesareans. Consistent with past recommendations, most women with one previous cesarean delivery with a low-transverse incision are candidates for and should be counselled about VBAC and offered a TOLAC. ${ }^{4}$

\section{Overall benefits}

A VBAC avoids major abdominal surgery, lowers a woman's risk of haemorrhage and infection, and shortens postpartum recovery. A successful VBAC has fewer complications than an elective repeat cesarean while a failed TOLAC has more complications than an elective repeat cesarean. ${ }^{4}$

\section{Objective}

To study the predictive factors for successful trial of labour after caesarean section (TOLAC) and to examine the risks and benefits involved in trial of labour in previous one low caesarean section.

\section{METHODS}

From a prospective study done at our medical college, we selected 75 cases with one prior low transverse cesarean delivery who wished to attempt a VBAC in the current pregnancy. TOLAC is routinely offered at our hospital to women meeting the standard criteria for a TOLAC, according to departmental protocol. After informed consent, the women included in this study were subjected to a thorough history to detect maternal and obstetric characteristics and a standard examination to estimate fetal weight, engagement of the fetal head, intra-partum features of fetal membranes, and cervical dilatation. After delivery, data on duration of labour, mode of delivery, birth outcome, and neonatal intensive care (NICU) admission were recorded and analysed. Specific inclusion and exclusion criteria were developed to determine study eligibility in terms of predictive factors. The inclusion criteria included singleton pregnancy with cephalic presentation, previous one low transverse caesarean section, non-recurrent indication, clinically estimated fetal weight $\leq 3.5 \mathrm{~kg}$, adequate pelvis on clinical assessment), less than or equal to 40 weeks and those willing to undergo a trial of scar. The exclusion criteria included gestational age <34 weeks, refusal for consent, associated high risk factors like those with severe medical disorders (severe hypertension, uncontrolled diabetes, or acute liver disorder), previous C.S. with history of complications ,contracted pelvis and delivery conception $<18$ months. This study was conducted after getting permission from institutional ethical committee. Proper counselling was done to the selected cases regarding risks and complications associated with TOLAC and also the benefits of VBAC. Informed consent was taken.

\section{RESULTS}

A total of 75 cases that were eligible for TOLAC, were included in our study protocol. Out of these, 32 cases underwent Elective repeat caesarean delivery (ERCD). The rest of 43 cases were given trial of labour, out of which 21 had successful VBAC and rest 22 underwent repeat caesarean section (RPCS).

Table 1: Mode of delivery in previous caesarean section cases.

\begin{tabular}{|l|l|l|}
\hline Mode of delivery & No. of cases & \% Out of total \\
\hline Total studied cases & 75 & \\
\hline ERCD & 32 & $42.7 \%$ \\
\hline TOLAC & 43 & $57.3 \%$ \\
\hline VBAC & 21 & $28 \%$ \\
\hline RPCS & 22 & $29.3 \%$ \\
\hline
\end{tabular}

This table shows that $57.3 \%$ (43 out of 75$)$ of total included cases underwent trial of labour after caesarean section (TOLAC) and rest 42.7\% (32 out of 75) ended up in Elective repeat caesarean delivery (ERCD).

Table 2: Age factor.

\begin{tabular}{|llllll|}
\hline Age (YRS) & $20-25$ & $25-30$ & $30-35$ & $35-40$ & Total \\
\hline ERCD & 6 & 10 & 13 & 3 & 32 \\
\hline VBAC & 9 & 11 & 1 & 0 & 21 \\
\hline RPCS & 6 & 7 & 8 & 1 & 22 \\
\hline
\end{tabular}

This table shows that maximum cases that attempted TOLAC and also, delivered successfully were of maternal age less than 30 years.

Table 3: Weeks of gestation at the time of delivery.

\begin{tabular}{|llll|ll|}
\hline $\begin{array}{l}\text { MGA } \\
\text { (weeks) }\end{array}$ & $36-37$ & $37-38$ & $38-39$ & $39-40$ & $\begin{array}{l}\text { Total } \\
\text { cases }\end{array}$ \\
\hline ERCD & 5 & 4 & 15 & 8 & 32 \\
\hline VBAC & 8 & 12 & 1 & 0 & 21 \\
\hline RPCS & 3 & 4 & 13 & 2 & 22 \\
\hline
\end{tabular}

This table shows that most cases $(95.2 \%)$ of VBAC delivered before 38 weeks of gestation, while most cases who underwent Caesarean section (either elective or repeat) were $>38$ weeks of gestation. 
Table 4: Comparison of obstetrical factors.

\begin{tabular}{|c|c|c|c|}
\hline \multirow[t]{2}{*}{ Factors } & \multirow[t]{2}{*}{$\begin{array}{l}\text { ERCD } \\
(32)\end{array}$} & \multicolumn{2}{|c|}{ TOLAC (43) } \\
\hline & & $\begin{array}{l}\text { VBAC } \\
\text { (21) }\end{array}$ & $\begin{array}{l}\text { RPCS } \\
(22)\end{array}$ \\
\hline $\begin{array}{l}\text { Estimated fetal } \\
\text { weight (EFW > } 3 \mathrm{~kg})\end{array}$ & $\begin{array}{l}22 \\
(68.75 \%)\end{array}$ & $4(19 \%)$ & $\begin{array}{l}10 \\
(45.4 \%)\end{array}$ \\
\hline $\begin{array}{l}\text { Cervical dilatation } \\
<4 \mathrm{~cm}\end{array}$ & $\begin{array}{l}32 \\
(100 \%)\end{array}$ & $2(9.5 \%)$ & $\begin{array}{l}4 \\
(18.1 \%)\end{array}$ \\
\hline $\begin{array}{l}\text { Fetal head station } \geq- \\
2\end{array}$ & $\begin{array}{l}28 \\
(87.5 \%)\end{array}$ & 0 & $\begin{array}{l}4 \\
(18.1 \%)\end{array}$ \\
\hline $\begin{array}{l}\text { Premature rupture of } \\
\text { membranes }\end{array}$ & $\begin{array}{l}18 \\
(56.25 \%)\end{array}$ & $\begin{array}{l}2 \\
(9.5 \%)\end{array}$ & $\begin{array}{l}12 \\
(54.5 \%)\end{array}$ \\
\hline $\begin{array}{l}\text { Prior vaginal } \\
\text { deliveries }\end{array}$ & 0 & $\begin{array}{l}6 \\
(28.5 \%)\end{array}$ & 0 \\
\hline
\end{tabular}

In our study, factors favouring VBAC were fetal weight $<3 \mathrm{~kg}$, fetal head engagement at the onset of labour, good bishop's score, with intact membranes, and prior vaginal deliveries. $81 \%$ VBAC cases delivered new born of less than equal to $3 \mathrm{~kg}$. In $90.5 \% \mathrm{VBAC}$ cases, cervical dilatation was $4 \mathrm{~cm}$ at the time of presenting in spontaneous labour and $28 \%$ cases had prior vaginal deliveries.

Table 5: Association of TOLAC cases with interval b/n 2 deliveries.

\begin{tabular}{|llllll|} 
& $1.5-3$ & $3-4.5$ & $4.5-6$ & $>6$ yrs & Total \\
& yrs & yrs & yrs & & \\
\hline VBAC & 3 & 10 & 3 & 5 & 21 \\
\hline RPCS & 8 & 6 & 4 & 4 & 22 \\
\hline
\end{tabular}

Out of all cases, $85.7 \%$ of VBAC cases had more than 3 years interval between previous C.S. and present delivery.

Table 6: Comparison of outcome of TOLAC with previous indication of caesarean section.

\begin{tabular}{|lll|}
\hline $\begin{array}{l}\text { Previous indication of } \\
\text { C.S. }\end{array}$ & \multicolumn{2}{l|}{ Outcome of TOLAC } \\
\hline & $\begin{array}{l}\text { VBAC } \\
\text { (out of 21) }\end{array}$ & $\begin{array}{l}\text { RPCS } \\
\text { (out of 22) }\end{array}$ \\
\hline Ante natal & $13(62 \%)$ & $8(36.3 \%)$ \\
\hline Breech & 7 & 1 \\
\hline Oligohydramnios & 2 & 4 \\
\hline Cord around neck & 1 & 1 \\
\hline Postdatism & 3 & 2 \\
\hline Intra-partum & $8(38 \%)$ & $14(63.7 \%)$ \\
\hline Fetal distress & 6 & 10 \\
\hline Non-progress of labour & 2 & 4 \\
\hline Total & $21(100 \%)$ & $22(100 \%)$ \\
\hline
\end{tabular}

This table compares the success of TOLAC with previous indication of caesarean section. VBAC happened in $62 \%$ of cases (13 out of 21) with ante natal indication, most common being breech (in 7 out of $21,53.8 \%$ cases) followed by post datism, oligohydramnios and cord around neck.

Table 7: Mode of delivery vs baby weight at birth.

\begin{tabular}{|ll|ll|}
\hline $\begin{array}{l}\text { Birth } \\
\text { weight }(\mathrm{kg})\end{array}$ & $\begin{array}{l}\text { VBAC } \\
\text { (out of 21) }\end{array}$ & $\begin{array}{l}\text { RPCS } \\
\text { (out of 22) }\end{array}$ & Total \\
\hline$<2.5$ & 4 & 3 & $7(10.71 \%)$ \\
\hline $2.5-3$ & 13 & 7 & $20(50 \%)$ \\
\hline $3-3.5$ & 4 & 10 & $14(39.29 \%)$ \\
\hline $3.5-4$ & 0 & 2 & 2 \\
\hline$>4$ & 0 & 0 & 0 \\
\hline
\end{tabular}

Most VBAC deliveries (17 out of 21 cases, $80.9 \%$ ) weighed less than $3 \mathrm{~kg}$. RPCS (12 out of 22 cases, $54.5 \%$ ) was done mostly in birth weight more than $3 \mathrm{~kg}$.

Table 8: indication of RPCS in unsuccessful TOLAC.

\begin{tabular}{|lll|}
\hline Indication & No. of RPCS & $\%$ of RPCS \\
\hline Non progress of labour & 6 & $27.2 \%$ \\
\hline Fetal distress & 7 & $31.8 \%$ \\
\hline Scar dehiscence & 9 & $41 \%$ \\
\hline Total & 22 & $100 \%$ \\
\hline
\end{tabular}

In cases of unsuccessful TOLAC cases, Repeat caesarean section (RPCS) was done for scar dehiscence (41\%), followed by fetal distress $(31.8 \%)$ and non progress of labour $(27.2 \%)$.

Vaccum delivery was performed in 4 (19\%) VBAC cases. Also, 1 case of uterine rupture noted.

\section{DISCUSSION}

Out of 75 cases included in our study, 43 cases $(57.3 \%$ of total cases) qualified for TOLAC during labour. Successful TOL (trial of labour) was observed in $48.8 \%$ (21 out of 43) resulting in VBAC (vaginal birth after caesarean) and the rest $51.2 \%$ of failed TOL ended in repeat caesarean delivery. In England, the overall cesarean delivery rate for 2012 to 2013 was $25.5 \%$; the majority of these deliveries were emergency (14.8\%), rather than elective $(10.7 \%)$, cesarean births. In the United States, the cesarean delivery rate was $31.3 \%$ in 2013, according to the American Congress of Obstetricians and Gynecologists. ${ }^{1}$

In our study, most of the women with successful VBAC were less than 30 years of age $(95.2 \%)$. Researchers found that younger women, aged 24 or less, were more likely to attempt a VBAC than women aged over 34, 60\% vs $45 \%$ respectively. Black women (62\%) and Asian women $(64 \%)$ were also found to have higher VBAC attempt rates for their second delivery when compared to white women $(49 \%) .^{5}$ Of the women who attempted a VBAC almost two-thirds (63\%) had a successful natural delivery. ${ }^{5}$ 
In our study, successful VBAC cases (90.5\% of cases) presented with cervical dilatation more than equal to 4 $\mathrm{cm}$. Cervical dilatation in the studied cases on admission was significantly higher in the successful TOLAC group compared to the unsuccessful TOLAC group, and the number of women admitted with cervical dilatation $<4$ $\mathrm{cm}$ was significantly higher in the unsuccessful TOLAC group; also, the duration of active labour was significantly lower in the successful TOLAC group. This was similar to findings reported in the literature; also, Durnwald et al, reported increased chances of a successful vaginal birth after cesarean section in women admitted with cervical dilatation of more than $1 \mathrm{~cm}^{6}$

In France in 2010, a trial of labour was attempted in $49 \%$ with $75 \%$ successful rate. The site of delivery does not appear to influence the rate of successful trial of labour. Two factors are strongly associated with vaginal birth after caesarean (VBAC): prior history of vaginal delivery and spontaneous labour. Many factors appear to decrease the rate of VBAC: maternal age above 40 years, body mass index greater than $30 \mathrm{~kg} / \mathrm{m}^{2}$, birth weights greater than $4000 \mathrm{~g}$, unfortunately, prediction of macrosomia seems to be inaccurate. ${ }^{7}$

In our study, most cases (95.2\%) of VBAC delivered before 38 weeks of gestation. Coassolo et al, reported a $31.3 \%$ TOLAC failure rate at 40 weeks or beyond, against $22 \%$ in $<40$ weeks. $^{8}$

Out of all VBAC's, 62\% (13 out of 21) had ante natal indication for their previous caesarean delivery; most common indication being breech followed by post datism and oligohydramnios.

In another study, the success rate of trial of labour was found to be $53.6 \%$. Higher chances of vaginal delivery were found with breech as an indication of previous caesarean section, i.e. $67.1 \%$ as compared to $39 \%$ with non-progress of labour as an indication. ${ }^{9}$

In our study, Repeat C.S. in failed TOL was performed for non-progress of labour (51\%) as the most common indication being scar dehiscence in $41 \%$ cases followed by fetal distress being the second common indication. The birth weight in most $(80.9 \%)$ cases of VBAC was $<=3 \mathrm{~kg}$. Vacuum was used in 4 cases of VBAC during $2^{\text {nd }}$ stage of labour.

Scar dehiscence was found in $1(0.9 \%)$ case of unsuccessful TOLAC, impending rupture was found in another case $(0.9 \%)$ of unsuccessful TOLAC, and the presence of premature rupture fetal membranes and/or use of oxytocin for augmentation does not affect the success of TOLAC in this study. Also, $0.2 \%-0.7 \%$ risk of scar dehiscence in women undergoing TOLAC was reported in the literature and by Cahill and colleagues. ${ }^{10,11}$

Uterine rupture is associated with an increased risk of severe maternal complications, such as hysterectomy, hemorrhage, as well as severe fetal complications, such as hypoxic ischemic encephalopathy and perinatal death". 12

The other important factor to consider in the setting of TOLAC or a uterine rupture is the neonatal outcomes. A large population-based study from Scotland determined that among 15,515 patients who underwent TOLAC, 7 perinatal deaths occurred as a result of uterine rupture, for a rate of 4.5 per 10,000 women. In comparison, there were no perinatal deaths due to uterine rupture among women undergoing elective repeat cesarean delivery, and the mortality rate was 0.5 per 10,000 among women laboring with no prior cesarean delivery. ${ }^{13}$

In our study, 1 case of fresh still birth was noted due to uterine rupture, NICU care was required in 5 cases of RPCS and 1 case of VBAC.

Ball et al. and Tan et al. reported increases in risks of neonatal morbidities and hypoxic ischemic encephalopathy (HIE) after an unsuccessful TOLAC. ${ }^{14,15}$ In this study, neonatal intensive care admission was significantly higher in the unsuccessful TOLAC group (2 due to birth asphyxia and 2 due to meconium aspiration and sepsis) compared to the successful group (1 case due to birth asphyxia).

If possible, avoid induction of labour, because induction of labour decreases the probability of success and increases the chance of uterine rupture in a trial of labour after cesarean delivery. Counsel patients who elect to undergo TOLAC to be evaluated early in labour and to manage the pregnancy in a hospital setting in which uterine rupture can be both recognized and managed expediently. ${ }^{16}$

Several investigators have found that "among women with prior cesarean delivery, the rates of maternal complications are highest among women who attempted vaginal birth and failed $(14.1 \%)$, intermediate among women who have a planned cesarean delivery (3.6\%), and the lowest among women who attempt vaginal birth and succeed (2.4\%)". 12,13

\section{CONCLUSION}

Trial of labour should be recommended to all women who have had only one previous cesarean section, except for: previous classical or $\mathrm{T}$ uterine incision, more than one cesareans has been performed, previous hysterotomy or full thickness myomectomy, previous uterine rupture, any contraindications to labour in this pregnancy (e.g. placenta previa, transverse lie, etc.), or if previous cesarean was performed for failure to progress in the active phase of labour.

However decisions should be made by physicians and patients on a case-by-case basis. If the patient is admitted in spontaneous labour, she should be evaluated by an 
obstetrician within 2 hours and be on continuous maternal and fetal monitoring.

In our study, $48.8 \%$ of women who were considered candidates for a trial of labour after cesarean (TOLAC) to attempt vaginal birth after cesarean (VBAC) had successful VBAC. Factors that increase the chances for a successful VBAC in an individual woman include:

- Younger age of women

- Lower gestational age at delivery

- Estimated fetal weight less than $3.5 \mathrm{~kg}$ at delivery

- A previous vaginal delivery, especially a previous VBAC

- Spontaneous onset of labour (labour is not induced)

- Normal progress of labour, including dilation and effacement (thinning) of the cervix

- Prior cesarean delivery performed because the baby's position was abnormal (breech)

- Only one prior cesarean delivery

- The prior cesarean delivery was performed early in labour, and not after full cervical dilatation.

Funding: No funding sources

Conflict of interest: None declared

Ethical approval: The study was approved by the Institutional Ethics Committee

\section{REFERENCES}

1. Updated VBAC Guidelines Released. Medscape. Oct 05, 2015 .

2. American College of Obstetricians and Gynecologists. ACOG Practice bulletin no. 115: Vaginal birth after previous cesarean delivery. Obstet Gynecol. 2010;116:450.

3. National Institutes of Health. National Institutes of Health Consensus Development Conference Statement vaginal birth after cesarean: new insights March 8-10, 2010. Semin Perinatol. 2010;34:351.

4. Practice Bulletin \#115, Vaginal Birth after Previous Cesarean Delivery, August 2010 issue of Obstetrics and Gynecology.

5. Knight HE, Gurol-Urganci I, van der Meulen JH, Mahmood TA, Richmond DH, Dougall A, et al. Vaginal birth after caesarean section: a cohort study investigating factors associated with its uptake and success. BJOG: An International Journal of Obstetrics and Gynaecology, 2013.
6. Durnwald C, Mercer B. Vaginal birth after cesarean delivery: Predicting success, risks of failure. J Matern Fetal Neonatal Med. 2004;15:388-93.

7. Predictive factors for vaginal birth after cesarean section. J Gynecol Obstet Biol Reprod (Paris). 2012;41(8):735-52.

8. Coassolo KM, Stamilio DM, Paré E, Peipert JF, Stevens E, Nelson DB, et al. Safety and efficacy of vaginal birth after cesarean attempts at or beyond 40 weeks of gestation. Obstet Gynecol. 2005;106:700-6.

9. Madaan M, Agrawal S, Nigam A, Aggarwal R, Trivedi SS. Trial of labour after previous caesarean section: the predictive factors affecting outcome. J Obstet Gynaecol. 2011;31(3):224-8.

10. Royal College of Obstetricians and Gynecologists (RCOG) Birth after previous caesarean birth. London (UK): Royal College of Obstetricians and Gynecologists (RCOG); (Green-top guideline; 45) 2007.

11. Cahill AG, Stamilio DM, Odibo AO, Peipert JF, Ratcliffe SJ, Stevens EJ. Is vaginal birth after cesarean (VBAC) or elective repeat cesarean safer in women with a prior vaginal delivery? Am J Obstet Gynecol. 2006;195:1143-7.

12. Smith GC, White IR, Pell JP, Dobbie R. Predicting cesarean section and uterine rupture among women attempting vaginal birth after prior cesarean section. PLoS Med. 2005;2(9):e252.

13. Landon MB, Hauth JC, Leveno KJ, Spong CY, Leindecker S, Varner MW. Maternal and perinatal outcomes associated with a trial of labor after prior cesarean delivery. N Engl J Med. 2004;351(25):2581-9.

14. Ball E, Hinshaw K. The current management of vaginal birth after previous cesarean delivery. The Obstetrian and Gynecologist. 2007;9:77-82.

15. Tan PC, Subramaniam RN, Omar SZ. Labor and perinatal outcome in women at term with one previous lower-segment Cesarean: A review of 1000 consecutive cases. ANZJ Obstet Gynaecol. 2007;47(1):31-6.

16. Studsgaard A, Skorstengaard M, Glavind J, Hvidman L, Uldbjerg N. Trial of labor compared to repeat cesarean section in women with no other risk factors than a prior cesarean delivery. Acta Obstet Gynecol Scand. 2013.

Cite this article as: Singh PS, Bamaniya J, Shah A. Factors predicting success of trial of labour after caesarean in current scenario. Int J Reprod Contracept Obstet Gynecol 2016;5:3893-7. 